\title{
Solving the speckle decorrelation challenge in acousto-optic sensing using tandem nanosecond pulses within the ultrasound period
}

\author{
Steffen Resink, Erwin Hondebrink, and Wiendelt Steenbergen* \\ Biomedical Photonic Imaging Group, MIRA Institute for Biomedical Technology and Technical Medicine, \\ University of Twente, P.O. Box 217, 7500 AE Enschede, The Netherlands \\ *Corresponding author: w.steenbergen@utwente.nl
}

Received September 3, 2014; accepted October 4, 2014;

posted October 17, 2014 (Doc. ID 222470); published November 11, 2014

\begin{abstract}
We present a novel acousto-optic (AO) method, based on a nanosecond laser system, which will enable us to obtain AO signals in liquid turbid media. By diverting part of the light in a delay line, we inject tandem pulses with $27 \mathrm{~ns}$ separation. The change of the speckle pattern, caused by the ultrasound phase shift, reduces the speckle contrast of the integrated speckle pattern captured in a single camera frame. With these tandem pulses, we were able to perform $\mathrm{AO}$ on a $2 \mathrm{~cm}$ liquid turbid medium in transmission mode. We show the raw signal and a spatial AO scan of a homogenous water-intralipid sample. This approach is potentially capable of AO probing in vivo, since the acquisition time (of approximately $40 \mathrm{~ns}$ ) is four orders of magnitude less than the typical time scales of speckle decorrelation found in vivo. The method may eventually enable us to obtain fluence compensated photoacoustic signals generated by the same laser. (c) 2014 Optical Society of America

OCIS codes: (170.1065) Acousto-optics; (030.6140) Speckle.
\end{abstract}

http://dx.doi.org/10.1364/OL.39.006486

Photoacoustic (PA) imaging currently finds many in vivo applications, where the optical absorption delivers the contrast. A major challenge toward quantification of optical absorption is to compensate the signal strength of PAs for local fluence variations. Recently, fluence compensation of PA signals was proposed by using acoustooptic (AO) imaging [1,2], in combination with PA [3]. This method relies on the principle that both $\mathrm{PA}$ and AO signal strength depend on the local fluence. The fluence dependency cancels out, when the PA signals are normalized by the AO signal.

To enable biomedical use, both PA and AO must work in living, dynamic tissues. While this is not a problem for $\mathrm{PA}$, for $\mathrm{AO}$, tissue dynamics has impeded practical in vivo application, based on spatial speckle analysis. $\mathrm{AO}$ detection is based on either analysis of dynamic laser speckles, or narrow band spectral filters, like spectral hole burning [4] and Fabry-Perot etalons [5]. Specklebased techniques are widely used, but suffer from speckle decorrelation, leading to long measurement times, and low signal-to-noise ratios. Ideally, a single AO measurement must be completed within the speckle decorrelation time. The general rule is that in tissues, the speckle decorrelation time is less than $1 \mathrm{~ms}$ [6], or even less than $0.1 \mathrm{~ms}$ [?]].

Recently, we showed the usability of high-intensity nanosecond pulsed lasers for AO signal generation [8], without the need of integrating over many pulses, as in other nanosecond pulsed AO methods, i.e., [9]. For overcoming stochastic variations in speckle contrast, some averaging is beneficial. The use of nanosecond pulses has two benefits. First of all, a pulsed laser easily delivers enough light within the speckle decorrelation time, so a bright speckle pattern of high contrast can be generated, if allowed by the laser line width. Second, nanosecond pulsed lasers are compatible with PAs, where this type of laser is typically used. While our previous method could only be applied in media with a decorrelation time much larger than the pulse interval time (often 50 $100 \mathrm{~ms}$ ), in this Letter we present a method that enables acquisition of an $\mathrm{AO}$ signal in scattering liquid samples, in approximately $40 \mathrm{~ns}$, hence far within the speckle decorrelation time of highly scattering liquids and biological tissues. This will enable in vivo fluence-compensated PA imaging, by adding AO probing using the same laser.

In speckle-based methods for AO tissue probing, ultrasound (US)-mediated intensity modulation of one or more speckles is used. The speckle intensity is modulated, with mainly the US frequency. The fraction of the light that shows this modulation is said to be "tagged," or "labeled," by US. For small modulation, this fraction is equal to the reduction in contrast [10]. Our recently presented method of AO signal detection [8] uses two pulses of several nanoseconds, each at a different phase of the US, such that the speckle pattern generated by the second pulse is slightly altered by the changed US interaction with the medium, compared to the first speckle pattern. Both speckle patterns were captured in two consecutive camera frames, and the AO effect was measured by either adding both images (and taking the speckle contrast reduction), or by subtracting them (and taking the standard deviation). In the concept presented in this Letter, we inject two laser pulses, with only tens of nanoseconds separation. On this time scale, there is virtually no speckle decorrelation, e.g., by Brownian motion or particle flow. The two pulses create two speckle patterns, I1 and I2, for which we can write

$$
I_{i}=\left\langle I_{i}\right\rangle+\Delta_{i},
$$

where \langle\rangle denotes spatial averaging over the detector surface, and $\Delta_{i}$ denotes a spatial perturbation from that average. Both patterns are normalized with $\left\langle I_{i}\right\rangle$ such that their average values are unity, hence 


$$
I_{i}=1+\Delta_{i}
$$

The speckle contrast $C$ is defined as

$$
C=\frac{\sigma}{\langle I\rangle}
$$

where $\sigma$ is the standard deviation, and $\langle I\rangle$ is the average value of the intensity of the speckle pattern. The theoretical maximum value for the speckle contrast is 1 , but several aspects are lowering the contrast of observed speckle patterns, such as the finite camera pixel size, the presence of two polarizations, and camera noise. For the maximum speckle contrast that can be obtained with this setup, from a single laser pulse, we write

$$
C_{i}^{2}=\left\langle\Delta_{i}^{2}\right\rangle=C_{\max }^{2}
$$

The camera adds the two speckle patterns in one exposure. Using Eqs. (2) and (4), the resulting speckle contrast is calculated as

$$
\begin{aligned}
C_{\text {sum }}^{2} & =\frac{\left\langle\left(I_{1}+I_{2}\right)^{2}\right\rangle}{4}-1 \\
& =\frac{1}{4}\left(\left\langle\Delta_{1}^{2}\right\rangle+\left\langle\Delta_{2}^{2}\right\rangle\right)+\frac{1}{2}\left\langle\Delta_{1} \Delta_{2}\right\rangle=\frac{1}{2} C_{\max }^{2}+\frac{1}{2} X,
\end{aligned}
$$

with $X=\left\langle\Delta_{1} \Delta_{2}\right\rangle$. The lower this correlation, the lower the speckle contrast, and two independent speckle patterns give $X=0$ and $C_{\text {sum }}=C_{\max } / \sqrt{2}$. The quantity that we use for quantifying the $\mathrm{AO}$ effect is the normalized reduction in speckle contrast

$$
\Delta C_{\text {norm }} \equiv \frac{C_{\max }-C_{\mathrm{sum}}}{C_{\max }} .
$$

Substituting Eq. (ㅁ) and rewriting Eq. (ㅁ), we obtain

$$
\Delta C_{\mathrm{norm}}=1-\sqrt{\frac{1}{2}+\frac{X}{2 C_{\max }^{2}}} .
$$

Taylor expansion of Eq. (7) around $X / C_{\max }^{2}=1$ gives

$$
\Delta C_{\text {norm }} \approx \frac{1}{4}-\frac{X}{4 C_{\max }^{2}} .
$$

When both patterns are identical, we have $X=C_{\max }^{2}$, making $\Delta C_{\text {norm }}$ zero. When the two initial speckle patterns slightly differ through the interaction of US with the medium, the correlation $X$ drops, and $\Delta C_{\text {norm }}$ increases. This method is the contrast method [10], with two short light pulses at different phases of the US, instead of a quasi-CW illumination.

We use $532 \mathrm{~nm}$ light of a frequency-doubled injection seeded Nd:YAG laser, with a pulse repetition rate of $10 \mathrm{~Hz}$ and a pulse energy of $350 \mathrm{~mJ}$ (Newport Quanta Ray lab series 170). Injection seeding of pulses, with a duration of $5 \mathrm{~ns}$, results in a coherence length of $1.5 \mathrm{~m}$. The laser is triggered by two synchronized function generators (Tektronix AFG 3102), and its pulses are attenuated with a half-wave plate and a polarizing beam splitter.

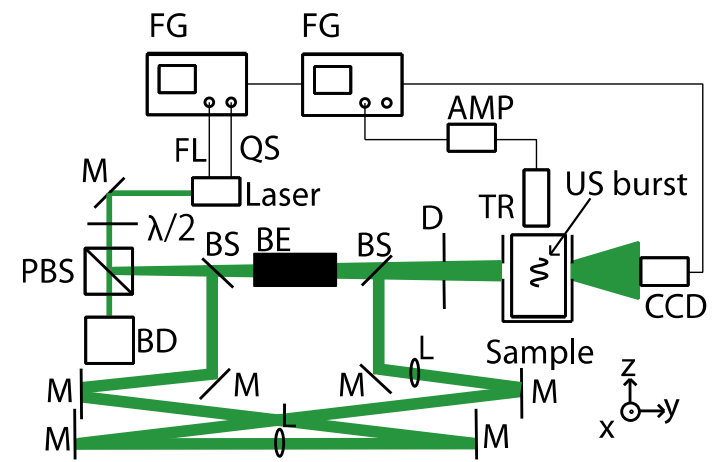

Fig. 1. Experimental setup; AMP, amplifier; BD, beam dump; $\mathrm{BE}$, beam expander; BS, 50:50 beamsplitter; D, diaphragm; FG, function generator; FL, flash lamp trigger of laser; L, lens $(f=1000 \mathrm{~mm})$; M, mirror; PBS, polarizing beam splitter; QS, $Q$-switch trigger of laser; TR, US transducer.

This system delivers enough light, in a single pulse, to generate a bright speckle pattern at the CCD camera (Allied Vision Technologies Manta G-145B NIR), after transmission through a turbid medium. The laser beam is split into two arms with a 50:50 beam splitter as shown in Fig. 1. The short arm $(30 \mathrm{~cm})$ leads to a second 50:50 beam splitter (that is used as a combiner); the second arm is extended with mirrors, to achieve a total length of $8 \mathrm{~m}$. This length difference causes a pulse delay of $27 \mathrm{~ns}$. The measured dual pulse light intensity signal is shown in Fig. 2. The benefit of a tandem pulse, versus a long pulse, is that all the light is only present at the extreme phases of the US. Furthermore, a long pulse does not provide stress confinement for PA applications, like a tandem pulse does with its small temporal features.

To compensate for beam divergence, we placed two lenses $(f=1000 \mathrm{~mm}$ ) along the delay line.

Without US modulation, the speckle patterns generated by the direct and delayed pulses should be identical. This requires a large spatial coherence between the two pulses, on the medium's surface. Due to the difference in propagation length, from the laser cavity and the applied lenses, the different curvatures of the phase fronts of both beams will compromise spatial coherence. To equalize the curvature of the phase fronts, a beam expander ( $f=-30$ and $f=75 \mathrm{~mm}$ ) is placed in the short arm. After the beam combiner, a diaphragm $(D=2 \mathrm{~mm})$ is placed for alignment purposes, and improving the beam quality. The amount of light reaching the sample is approximately $3 \mathrm{~mJ}$ per tandem pulse. We use our setup

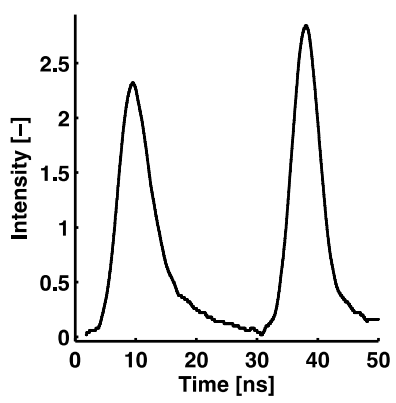

Fig. 2. Temporal profile of the tandem pulse, resulting from the $8 \mathrm{~m}$ delay line, measured with a photodiode with a rise time less than $1 \mathrm{~ns}$. 
in transmission mode, and the camera that captures the speckle patterns is placed $15 \mathrm{~cm}$ behind the $\varnothing 3 \mathrm{~mm}$ rear aperture of the sample. The wave fronts on the medium's surface of the fast and slow pulses must be very similar in curvature, angle, and intensity distribution for this method to work. To test this, we compared the speckle pattern when only the short path is used, with a speckle pattern when both paths are used. If the wave front curvature or intensity distribution is different, the integrated speckle pattern will have a reduced contrast. When the angle of incidence is not matched properly, then the long path light will generate a translated copy of the speckle pattern, formed by the short path light for thin samples (the "memory effect" [11]). These "shifted" patterns are the input for the next thin layer of the sample; this also results in a lower contrast of the integrated speckle pattern. Figure 3 shows that the contrast for a speckle pattern, by using only the short arm of the setup, is 0.545 ; when both arms are used, the resulting contrast is 0.528 . This reduction of $3 \%$ is sufficiently small, because close to $90 \%$ of the potential maximum signal strength (which is a contrast reduction from 0.5 to 0.25 ) is still available. In other words, the difference between zero signal and maximum signal is $12 \%$ smaller than optimal. Furthermore, the speckle contrast reduction has a big stable component; only the variation of the speckle contrast reduction is a source of noise in the measurement.

To create US modulation, the waveform from the function generator is a sine at $5 \mathrm{MHz}$, with 15 cycles. The waveform signal is amplified by $\sim 50 \mathrm{~dB}$ by the amplifier (Electronics \& Innovation A075), whose output is connected to a focused $\left(f=22.3 \mathrm{~mm}, D_{\text {focus }}=1.1 \mathrm{~mm}\right)$ $5 \mathrm{MHz}$ US transducer (Olympus Panametrics-NDT V310).

The sample consists of a $20 \mathrm{~mm}$ diameter cylinder, which is filled with a homogeneous mixture [3\% intralipid $20 \%\left(\mu \mathrm{s}^{\prime} \approx 5 \mathrm{~cm}^{-1}\right)$ and water]. Based on [12], we estimate the speckle decorrelation time to be approximately $25 \mu \mathrm{s}$.

In a first experiment, we toggled between an US "on" and US "off" state. The speckle contrast reduction $\left(\Delta C_{\text {norm }}\right)$, relative to a reference speckle pattern captured at the start of the experiment, is plotted.

In a second experiment, we performed a scan through the same homogeneous sample, to obtain the so-called banana shape, which is the normalization parameter in sound light, where fine structural information is obtained using PA. A focused US burst of 15 cycles scans the $x y$ plane at the optical axis, by mechanical translation at a step size of $0.5 \mathrm{~mm}$. The sample is illuminated by tandem pulses, when the US burst intersects the $x-y$ plane.
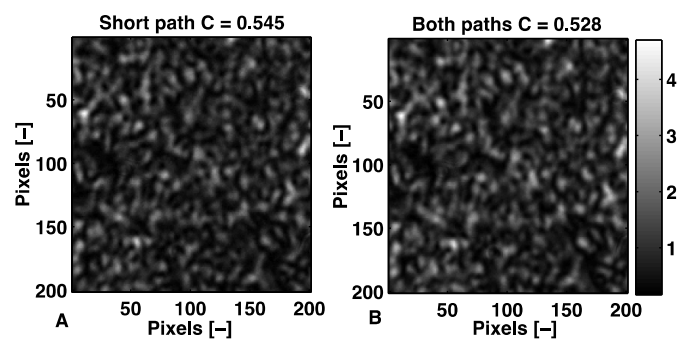

Fig. 3. Normalized speckle patterns for light transmitted through paper; (a) when only the short arm is used; (b) when both paths are used.

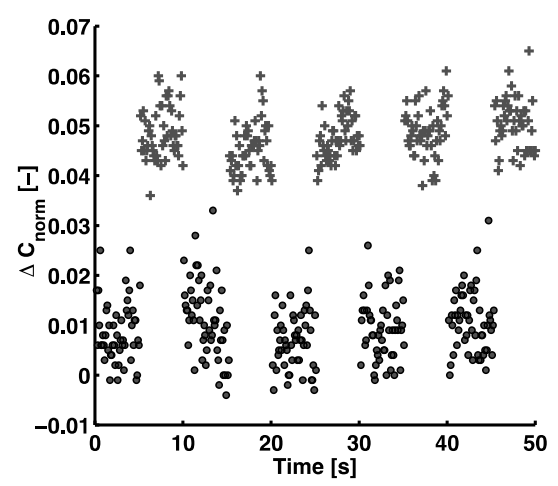

Fig. 4. Raw AO signal obtained with the setup, with US applied $(+)$, interleaved with the background, when no US is applied (O). Each image point is generated by one pulse pair, separated by $27 \mathrm{~ns}$.

In Fig. 4, the raw AO signal is plotted when the sound is switched off and on $5 \times$. The signal during the US "on" episodes is increased, hence the speckle contrast reduced. The average signal strength, defined as the difference of the averages in the "on" and "off" stages, was 0.039 , with an RMS value of 0.0066 during the off stages, hence the SNR we obtained was approximately 6 .

In the second experiment, the $x y$-distribution of $\Delta C_{\text {norm }}$ was measured $6 \times$, and the median value is displayed in Fig. 5. The apertures for light injection and reception are at the top and bottom, respectively. As expected, the signal strength along the optical axis was the highest, and lower to the sides.

We clearly observe an $\mathrm{AO}$ signal with our setup in a $2 \mathrm{~cm}$ liquid sample, with the AO signal obtained in $27 \mathrm{~ns}$ only. An AO signal was measured in [13] in a waterintralipid mixture, based on the autocorrelation of the signal from a photomultiplier tube, capturing the light of a single speckle. Their acquisition time was between 0.5 and $5 \mathrm{~s}$. Assuming a speckle decorrelation time of $25 \mu \mathrm{s}$, they sampled approximately $2 \times 10^{4}$ to $2 \times 10^{5}$ statistically independent speckles within that acquisition time. This is roughly the same number as we sample in parallel with our camera, in $27 \mathrm{~ns}$. Therefore, we expect the same noise level, but for a much shorter acquisition time. Other speckle pattern based methods, like holography $[14,15]$, might be able to show better AO signals, but require illumination over a full US cycle. Furthermore, the fluences required for deep PA imaging might damage the AO modulators in the reference arm.

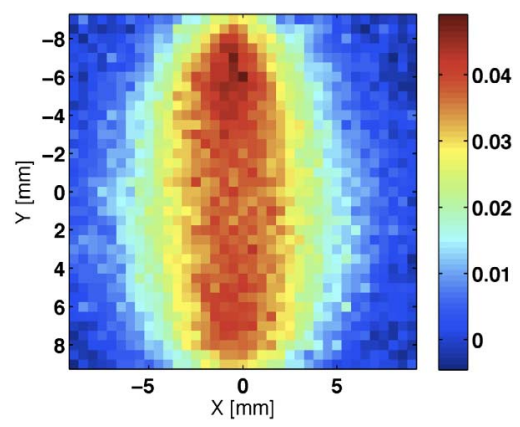

Fig. 5. Distribution of $\Delta C_{\text {norm }}$, measured in the $x-y$ plane, in a liquid scattering phantom. 
The AO scan shown in Fig. 5 clearly shows a "bananashaped" radiance distribution of photons, travelling from the injection to the exit aperture. The relatively low SNR of 6 has several causes. First, the 27 ns time delay between the two pulses is not optimal. The best delay time would be half the US period [8], or $100 \mathrm{~ns}$ for $5 \mathrm{MHz}$ US, potentially leading to a fourfold increase in SNR. However, a longer delay line would pick up more distortions from small density fluctuations in the air, extra mirror surfaces, and lenses. Furthermore, the imperfect spatial coherence of both pulses, at the entrance plane, reduces the signal. Without US, both pulses should ideally generate exactly the same speckle pattern. However, when the wave fronts of both paths do not exactly match, the speckle patterns are different. These small deviations from the ideal situation result in a lowered contrast of the combined speckle pattern. Additional changes induced by the US reduce the contrast less. The normalization with $C_{\max }$ corrects for this effect; however, it amplifies both the noise and signal. Therefore, changes in the design of the delay line should aim for a higher spatial coherence between the two pulses, along with a longer delay time.

The values in the scan displayed in Fig. 5 appear to be slightly lower than observed in the raw signal, shown in Fig. 4. This is due to the use of a different reference speckle pattern, and application of the median filter, that removes the extreme values of the noise.

The presented method solves the challenges posed by speckle decorrelation in dynamic samples. Furthermore, the pulse energy and pulse duration used in the research reported in this Letter also allow for PA signal generation. The small time delay between the two light pulses ensures that only high-frequency PA signals will be affected by the double pulse excitation. Combined with the pulse energies of several $\mathrm{mJ}$, this implies that our setup is also adequate for typical $1-5 \mathrm{MHz}$ PA signals. This is an important step toward in vivo fluencecompensated PA imaging, by adding AO probing using the same laser.

This Letter was supported by the Foundation for Fundamental Research on Matter (FOM), which is part of the Netherlands Organization for Scientific Research (NWO), under Grant 09NIG01.

\section{References}

1. D. S. Elson, R. Li, C. Dunsby, R. Eckersley, and M. X. Tang, Interface Focus 1, 632 (2011).

2. S. G. Resink, A. C. Boccara, and W. Steenbergen, J. Biomed. Opt. 17, 040901 (2012).

3. K. Daoudi, A. Hussain, E. Hondebrink, and W. Steenbergen, Opt. Express 20, 14117 (2012).

4. Y. Z. Li, P. Hemmer, C. H. Kim, H. L. Zhang, and L. H. V. Wang, Opt. Express 16, 14862 (2008).

5. S. Sakadzic and L. H. V. Wang, Opt. Lett. 29, 2770 (2004).

6. L. H. V. Wang, Dis. Markers 19, 123 (2003).

7. M. Lesaffre, F. Jean, F. Ramaz, A. C. Boccara, M. Gross, P. Delaye, and G. Roosen, Opt. Express 15, 1030 (2007).

8. S. G. Resink, E. Hondebrink, and W. Steenbergen, Opt. Express 22, 3564 (2014).

9. B. Judkewitz, Y. M. Wang, R. Horstmeyer, A. Mathy, and C. H. Yang, Nat. Photonics 7, 300 (2013).

10. J. Li, G. Ku, and L. H. V. Wang, Appl. Opt. 41, 6030 (2002).

11. I. Freund, M. Rosenbluh, and S. Feng, Phys. Rev. Lett. 61, 2328 (1988).

12. A. Bratchenia, "Towards quantitative acoustic-optic imaging," Ph.D. dissertation (University of Twente, Enschede, The Netherlands, 2010), p. 148.

13. A. Lev and B. Sfez, J. Opt. Soc. Am. A 20, 2347 (2003).

14. M. Atlan, B. C. Forget, F. Ramaz, A. C. Boccara, and M. Gross, Opt. Lett. 30, 1360 (2005).

15. K. Daoudi, A. C. Boccara, and E. Bossy, Appl. Phys. Lett. 94, 154103 (2009). 